\title{
Periodic Pattern Analysis Under Affine Distortions Using Wallpaper Groups
}

\author{
Yanxi Liu and Robert T. Collins \\ The Robotics Institute, Carnegie Mellon University, Pittsburgh 15213, USA, \\ yanxi,rcollinsecs.cmu.edu, \\ http://www.cs.cmu . edu/ yanxi/www/home.html
}

\begin{abstract}
In this paper, the mathematical theory of wallpaper groups is used to construct a computational tool for symmetry analysis of periodic patterns. Starting with a novel peak detection algorithm based on "regions of dominance", an input periodic pattern can be automatically classified into one of the 17 wallpaper groups. The orbits of stabilizer subgroups within the group lead to a small set of candidate motifs that exhibit local symmetry consistent with the global symmetry of the entire pattern. We further consider affine distorted periodic patterns and show that each such pattern can be classified into a small set of symmetry groups that describe the patterns' potential symmetries under affine transformation.
\end{abstract}

\section{Introduction}

Symmetry is pervasive in both natural and man-made environments. Humans have an innate ability to perceive symmetry, but it is not obvious how to automate this powerful insight. It is a continuous effort of the authors to find proper computational tools for dealing with symmetry. Symmetries of periodic patterns in a plane are of particular interest in computer vision. This is because the symmetry group of a pattern is independent of scale, absolute color, lighting, density and orientation/position of the pattern. Periodic patterns can be found in regular textures, indoor and outdoor scenes (e.g. brick walls, tiled floors, wallpapers, ceilings, clothes, windows on buildings, cars in a parking lot), or in intermediate data representations (e.g. periodicity analysis of human and animal gaits in the spatio-temporal domain).

A mature mathematical theory for periodic patterns has been known for over a century [1,2]. For monochrome planar periodic patterns, there are seven frieze groups for $2 \mathrm{D}$ patterns repeated along one dimension, and seventeen wallpaper groups describing patterns extended by two linearly independent translational generators. Despite an infinite variety of instantiations, this finite set of symmetry groups completely characterizes the possible structural symmetry of any periodic pattern.

We have developed a computational model of periodic pattern perception composed of: generating the underlying translational lattice from the image of a periodic pattern, classifying the symmetry group of the periodic pattern, and 
identifying the preferred "motif" of the pattern. Our work, initially inspired by [7], appears to be the first to use the theory of frieze and wallpaper groups for automated analysis of periodic patterns, although there exist flowcharts and computer programs that allow humans to interactively generate and identify periodic patterns for educational purposes $[8,3]$. Due to space limitations, this paper concentrates only on wallpaper groups. Furthermore, we assume that the translational lattice of the $2 \mathrm{D}$ periodic pattern has already been extracted. The reader is referred to [5] to find our algorithm for performing robust lattice extraction. Figure 1 shows one sample result produced by this algorithm.

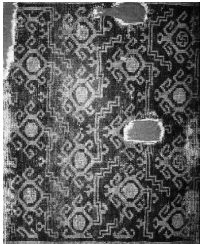

rug

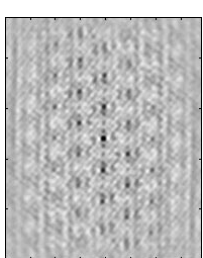

(A)

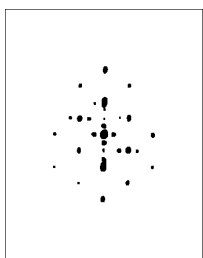

(B)

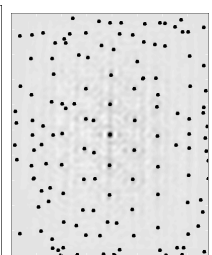

(C)

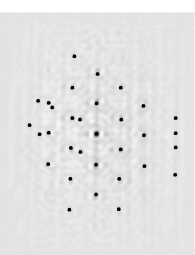

(D)

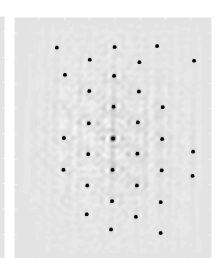

(E)

Fig. 1. An oriental rug image and A) its autocorrelation surface, B) peaks found using a global threshold, C) peaks extracted using the threshold-free method of Lin, et al. [4], D) the highest 32 peaks from those returned by Lin, et al., E) the 32 most-dominant peaks found using our approach described in [5].

\section{Symmetry Group Classification under Euclidean Transformations}

A 2D repeated or periodic pattern has the following property: there exists a finite region bounded by two linearly independent translations which, when acted upon by the group generated by the translations, produces simultaneously a covering (no gaps) and a packing (no overlaps) of the original image [7,2]. The smallest such bounded region is called a unit of the pattern or lattice unit, since the translational orbit of any single point on the plane is a lattice. A symmetry of a subset $S$ of Euclidean space is an isometry that keeps $S$ setwise invariant. All symmetries of $S$ form the symmetry group of $S$ under composition. It has been proven that there are seventeen wallpaper groups (Figure 2) describing patterns extended by two linearly independent translational generators $[7,2]$. Mathematically, wallpaper groups are defined only for infinite patterns that cover the whole plane. In practice, we analyze a periodic pattern $P$ of a finite area, and use the phrase "symmetry group $G$ of $P$ " to mean that $G$ is the symmetry group of the infinite periodic pattern that has $P$ as a finite patch.

Figure 2 depicts unit lattices for the 17 distinct wallpaper groups (from [7]). Each unit is characterized in terms of its translation generators, rotation, reflec- 

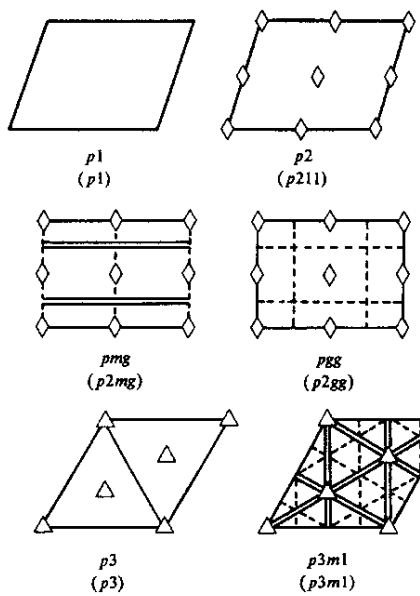

$\underset{(p 211)}{\rho 2}$
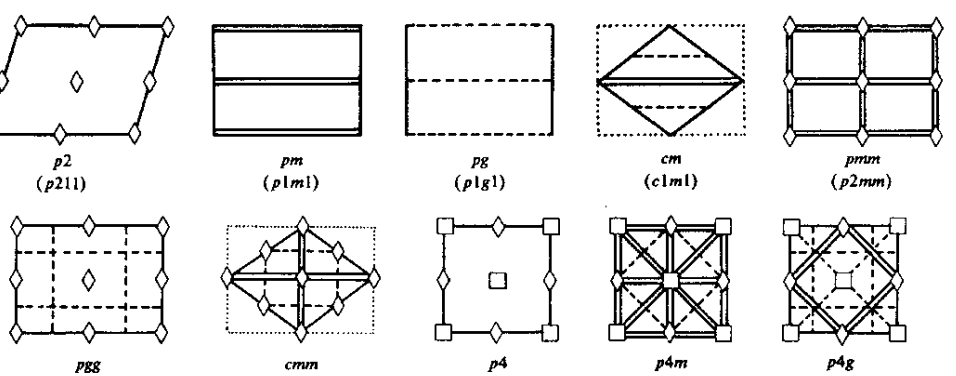

$\underset{\substack{p m \\(p|m|)}}{p}$ $\underset{\substack{p g \\(p \mid g 1)}}{p(1)}$

$\underset{(c \mid c m)}{c|m| 1)}$

(p2mmm)
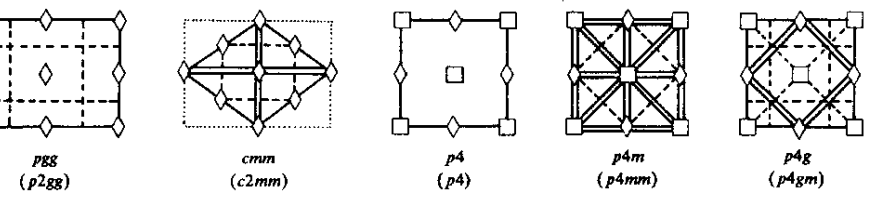

$(c 2 m m)$

(p4)
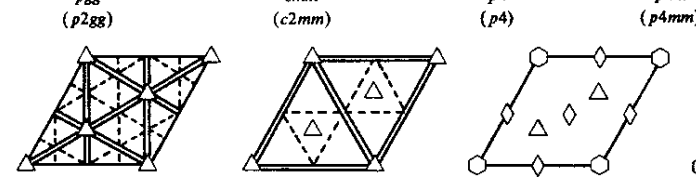

$p 3 m 1$
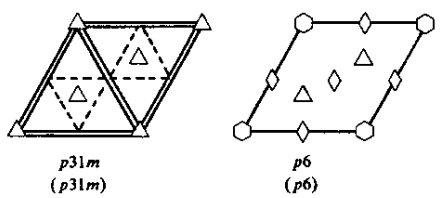

$p 6$

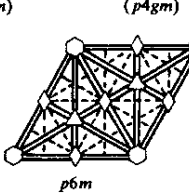

$p 3$
$(p 3)$

$(p 3 m 1)$

$(p 31 m)$

(p6)
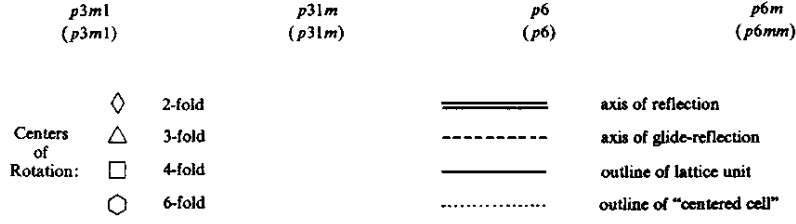

Fig. 2. The generating regions for the 17 Wallpaper groups (from [7])

tion and glide-reflection symmetries. The two linearly independent translations of minimum length are the two basic generators of each group, and they construct a lattice for the group. Even though the variety of pattern instantiations is endless, the underlying relationship between translation, rotation, reflection and glide-reflection in any $2 \mathrm{D}$ periodic pattern must conform to one of these seventeen cases.

Since a symmetry of a $2 \mathrm{D}$ periodic pattern has to map the lattice associated with the pattern onto itself, i.e., map centers of rotation to new centers of rotation having the same order, the only possible rotation symmetries are 2, 3, 4, 6-fold rotations. This restriction is often referred to as the crystallographic restriction. Furthermore, reflection axes can only be oriented parallel, diagonal, or perpendicular to the lattice translation vectors. Under these constraints, there are only five possible lattice unit shapes: (1) parallelogram (two groups: $p 1, p 2$ ), (2) rectangular (five groups: $p m, p g, p m m, p m g, p g g$ ), (3) rhombic (two groups: $\mathrm{cm}, \mathrm{cmm}$ ), (4) square (three groups: $p 4, p 4 m, p 4 g$ ) and (5) hexagonal (five groups: $p 3, p 3 \mathrm{ml}, p 3 \mathrm{~lm}, p 6, p 6 \mathrm{~m})$. All lattice units are parallelograms. Rectangular units have angles of $90^{\circ}$. Rhombic units have equal-length edges. Square units are a special case of both (2) and (3), and hexagonal units are a special case of (3).

We have constructed an algorithm that can automatically classify which symmetry group a 2D periodic pattern under Euclidean transformations belongs to. The practical value of understanding the 17 wallpaper groups is that correct pattern classification can be performed after verifying the existence of only a small 
set of rotation and/or reflection symmetries. Table 1 lists the eight symmetries checked in the classification algorithm. It is clear that each group corresponds to a unique sequence of values listed in Table 1 , and all are mutually exclusive from each other. The determination of a specific rotation or reflection or glidereflection symmetry is performed by applying the symmetry to be tested to the entire pattern, then checking the similarity between the original and transformed images.

Table 1. Wallpaper group classification: numbers $2,3,4$ or 6 denote n-fold rotational symmetry, Tx (or Dx) denotes reflectional symmetry about one of the translation (or diagonal) vectors of the unit lattice. "Y" means that the symmetry exists for that particular symmetry group; empty space means no. $\mathrm{Y}(\mathrm{g})$ denotes a glide reflection.

\begin{tabular}{|l|c|c|c|c|c|c|c|c|c|c|c|c|c|c|c|c|c|}
\hline & $\mathrm{p} 1$ & $\mathrm{p} 2$ & $\mathrm{pm}$ & $\mathrm{pg}$ & $\mathrm{cm}$ & $\mathrm{pmm}$ & $\mathrm{pmg}$ & $\mathrm{pgg}$ & $\mathrm{cmm}$ & $\mathrm{p} 4$ & $\mathrm{p} 4 \mathrm{~m}$ & $\mathrm{p} 4 \mathrm{~g}$ & $\mathrm{p} 3$ & $\mathrm{p} 3 \mathrm{~m} 1$ & $\mathrm{p} 31 \mathrm{~m}$ & $\mathrm{p} 6$ & $\mathrm{p} 6 \mathrm{~m}$ \\
\hline 2 & & $\mathrm{Y}$ & & & & $\mathrm{Y}$ & $\mathrm{Y}$ & $\mathrm{Y}$ & $\mathrm{Y}$ & $\mathrm{Y}$ & $\mathrm{Y}$ & $\mathrm{Y}$ & & & & $\mathrm{Y}$ & $\mathrm{Y}$ \\
\hline 3 & & & & & & & & & & & & & $\mathrm{Y}$ & $\mathrm{Y}$ & $\mathrm{Y}$ & $\mathrm{Y}$ & $\mathrm{Y}$ \\
\hline 4 & & & & & & & & & & $\mathrm{Y}$ & $\mathrm{Y}$ & $\mathrm{Y}$ & & & & & \\
\hline 6 & & & & & & & & & & & & & & & & $\mathrm{Y}$ & $\mathrm{Y}$ \\
\hline $\mathrm{T} 1$ & & & $\mathrm{Y}$ & $\mathrm{Y}(\mathrm{g})$ & & $\mathrm{Y}$ & $\mathrm{Y}(\mathrm{g})$ & $\mathrm{Y}(\mathrm{g})$ & & & $\mathrm{Y}$ & $\mathrm{Y}(\mathrm{g})$ & & & $\mathrm{Y}$ & & $\mathrm{Y}$ \\
\hline $\mathrm{T} 2$ & & & & & & $\mathrm{Y}$ & $\mathrm{Y}$ & $\mathrm{Y}(\mathrm{g})$ & & & $\mathrm{Y}$ & $\mathrm{Y}(\mathrm{g})$ & & & $\mathrm{Y}$ & & $\mathrm{Y}$ \\
\hline $\mathrm{D} 1$ & & & & & $\mathrm{Y}$ & & & & $\mathrm{Y}$ & & $\mathrm{Y}$ & $\mathrm{Y}$ & & $\mathrm{Y}$ & $\mathrm{Y}$ & & $\mathrm{Y}$ \\
\hline $\mathrm{D} 2$ & & & & & & & & & $\mathrm{Y}$ & & $\mathrm{Y}$ & $\mathrm{Y}$ & & & & & $\mathrm{Y}$ \\
\hline
\end{tabular}

\section{$3 \quad$ Extracting Representative Motifs}

Although other work has addressed detection of the translational lattice of a periodic pattern, ours is the first to seek a principled method for determining a representative motif. The issue here is that consideration of translational symmetry alone fixes the size, shape and orientation of the lattice, but leaves open the question of where the lattice is located in the image. Any offset of the lattice carves the pattern into a set of identical tiles, but these tiles typically provide no computational insight, and appear nonintuitive to a human observer (Figure 3). Choosing a good motif should help one see, from a single tile, what the whole pattern looks like. From work in perceptual grouping, it is known that the human perceptual system often has a preference for symmetric figures. Our contribution in this section is to show how a small set of tiles can be chosen, in a principled way, such that the symmetry of the pattern fragments on them is maximized.

If we entertain the idea that the most representative motif is the one that is most symmetrical, one plausible strategy for generating motifs is to align the motif center with the center of the highest-order of point symmetry in the pattern. This is the point fixed by the largest stabilizer subgroup of the symmetry group of the pattern. If we choose the centers of the highest order of rotational 


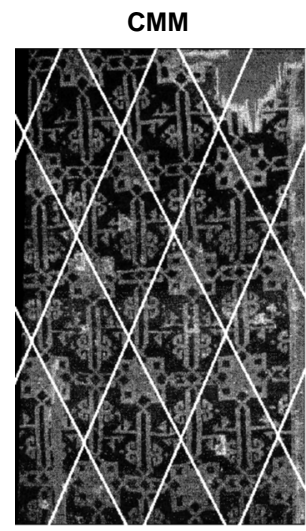

(A)

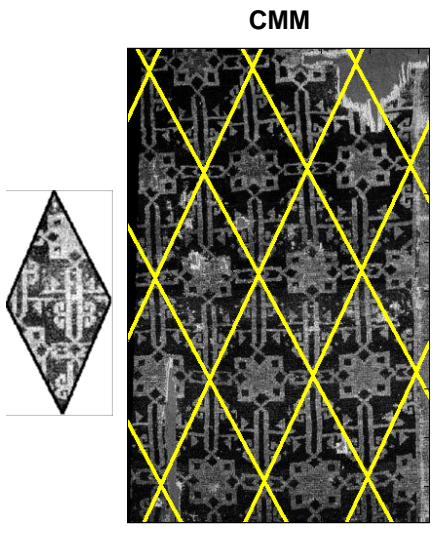

(C)

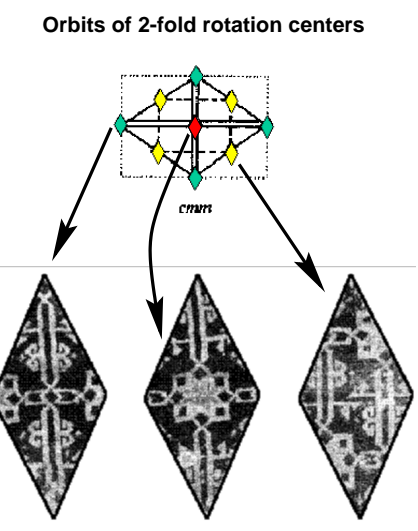

(D)

Fig. 3. (A) and (B) show an automatically extracted lattice and the tile that it implies. The tile is not a good representation of the pattern motif. (C) and (D) show the lattice position in terms of one of the three most-symmetric motifs found for the oriental rug image. The latter was generated automatically by an algorithm that analyzes pattern symmetry based on knowledge of the 17 wallpaper groups.

symmetries, candidate motifs can then be determined systematically by enumerating each distinct center point of the highest-order rotation. Two rotation centers are distinct if they lie in different orbits of the symmetry group, that is, if one cannot be mapped into the other by applying any translation, rotation, reflection or glide-reflection symmetries in its own symmetry group.

Figure 3 shows an example of an automatically extracted lattice, and an arbitrary tile that it carves out, followed by three symmetrical tiles centered on 2-fold rotation centers More examples and explanations can be found in [5].

\section{Symmetry Group Classification Under Affine Transformations}

When a 2D pattern undergoes a rigid transformation, its symmetry group remains. Strictly speaking, its symmetry group is conjugated by the transformation that acts on the pattern. Since there exists a bijection between the original symmetry group and the conjugated symmetry group, the two groups are considered equivalent (isomorphic). If one imagines a coordinate system fixed on the pattern, the translation, rotation, reflection and glide-reflection symmetries are unchanged under this coordinate system when the pattern is undergoing rigid transformations. This situation will no longer be true when the pattern undergoes a non-rigid transformation. However, certain symmetries of a periodic pattern may survive. 


\subsection{Wallpaper Group Transition Matrix}

If $g$ is a symmetry of a 2D periodic pattern $P$, by the definition of symmetry $g(P)=P$. Let $T(g(P))=T(P)$, here $T$ is a transformation. Then $T\left(g T^{-1} T(P)\right)=$ $T(P) \Rightarrow T g T^{-1}(T(P))=T(P)$. A useful question to ask is: Does $T g T^{-1}$ remain a symmetry of $T(P)$ ? The answers, of course, depend on what $g$ and $T$ are. The answer is "yes" if (1) $T$ is a similarity transformation (a proof that under similarity transformation, a periodic pattern remains the same in terms of its symmetry group) (2) $T$ is an affine transformation and $g$ is either a translation (a proof that a periodic pattern remains a periodic pattern under affine transformation) or $g$ is a 2 -fold rotation; (3) $g$ is a reflection (glide-reflection) and $T$ is a non-uniform scaling parallel or perpendicular to $g$ 's reflection axis. Relevant proofs can be found in [6]. Based on these proven results, we can construct a $17 \times 17$ wallpaper group transition matrix (Table 2) that dictates how the symmetry group of a periodic pattern can be transformed into other groups under non-rigid transformations. It turns out that only certain groups can be associated with a pattern under affine distortions. This matrix leads to a new way of evaluating a periodic pattern affine deformation: we should not only consider the symmetry group of the pattern as given, but also all the possible symmetry groups that can be associated with that pattern when it transformed affinely. Table 2 tells us that these transitions form well-defined small, finite orbits. For example, there are two large orbits of the 17 groups: the $p 1$-orbit and the $p 2$-orbit. This comes from the fact that 2 -fold rotation always survives any nonsingular affine distortion. Figure 4 shows one example of symmetry group transition as a pattern undergoes a series of affine deformations.

\subsection{Symmetry Group Classification Algorithm}

When the 2D pattern undergoes an affine transformation that preserves the shortest vector property, the same Euclidean algorithm (Table 1) can be applied for determining the lattice unit and classifying its symmetry group ${ }^{1}$. From Table 2, only those entries with $P$ need to be further checked for possible "higher symmetries".

The implementation of this idea is carried out as follows: Once the lattice unit is decided, the input unit lattice is simultaneously deformed into a hexagonal lattice and a square lattice, with the pattern deformed accordingly. Hexagonal and square lattices are the most symmetrical lattices, therefore these deformations allow the most symmetrical potential patterns to form. Meanwhile, the original symmetries of the pattern are guaranteed to be preserved under at least one of these two deformations, because hexagonal and square lattices are special cases of the more general lattices (rhombus, rectangular and parallelogram, see Section 2). The group classification procedure can then proceed in the same way

\footnotetext{
${ }^{1}$ When the affine distortion is so large that the nearest neighboring lattice points no longer form the boundary of a proper generating region, additional information is needed to locate the lattice unit. These include finding an axis of skewed symmetry, which is beyond scope of this paper.
} 
Table 2. Wallpaper Group Transition Matrix

Empty entries mean that there exist no transformations between the two groups (to the left and to the top). S: similarity transformation, $\mathrm{N}$ : non-uniform scaling $\perp$ or $\|$ to all reflection axes in the group to the left, A: general affine transformation other than $\mathbf{S}$ or $\mathbf{N}$, and $\mathbf{P}$ : possible affine transformation (pattern dependent).

\begin{tabular}{|l|l|l|l|l|l|l|l|l|l|l|l|l|l|l|l|l|l|}
\hline & p1 & p2 & pm & pg & cm & pmm & pmg & gg & cmm & p4 & p4m & p4g & p3 & p3m1 1 & p31m & $\mathrm{p} 6$ & $\mathrm{p} 6 \mathrm{~m}$ \\
\hline p1 & A & & P & P & P & & & & & & & & P & P & P & & \\
\hline p2 & & A & & & & P & P & P & P & P & P & P & & & & P & P \\
\hline pm & A & & N & & & & & & & & & & & & & & \\
\hline pg & A & & & N & & & & & & & & & & & & & \\
\hline cm & A & & & & N & & & & & & & & & P & P & & \\
\hline pmm & & A & & & & N & & & & & P & & & & & & \\
\hline pmg & & A & & & & & N & & & & & & & & & & \\
\hline pgg & & A & & & & & & N & & & & P & & & & & \\
\hline cmm & & A & & & & & & & N & & P & P & & & & & P \\
\hline p4 & & A & & & & & & & & S & & & & & & & \\
\hline p4m & & A & & & & N & & & N & & S & & & & & & \\
\hline p4g & & A & & & & & & N & N & & & S & & & & & \\
\hline p3 & A & & & & & & & & & & & & S & & & & \\
\hline p3ml & A & & & & N & & & & & & & & & S & & & \\
\hline p31m & A & & & & N & & & & & & & & & & S & & \\
\hline p6 & & A & & & & & & & & & & & & & & S & \\
\hline p6m & & A & & & & & & & N & & & & & & & & S \\
\hline
\end{tabular}

as stated in Section 2. A diagram version of the algorithm is shown in Figure 5.

\subsection{Symmetry Group Classification Experimental Results}

We have successfully processed all seventeen wallpaper group patterns ${ }^{2}$. Here we provide one example to illustrate how our algorithm works.

The first step is to determine the underlying translational lattice structure of the original image, in the form of two independent generating vectors $t_{1}$ and $t_{2}$. Since we are assuming that the wallpaper pattern has been previously isolated, the lattice points are determined by finding significant peaks in the pattern's autocorrelation surface (Figure 6a-c). The lattice of dots is decomposed into two generating vectors by finding the two shortest difference vectors $t_{1}$ and $t_{2}$ such that the angle between them is between 60 and 90 degrees. The second step involves transforming the lattice to a square grid, aligned with the horizontal and vertical axes (Figure 6d-f). This is performed by applying an affine transformation to the image and its autocorrelation surface. The transformation used is the unique affine transform leaving the origin $(0,0)$ fixed and taking $t_{1}$ to $(L, 0)$

\footnotetext{
${ }^{2}$ For a more complete set of results on all 17 wallpaper groups see [6].
} 


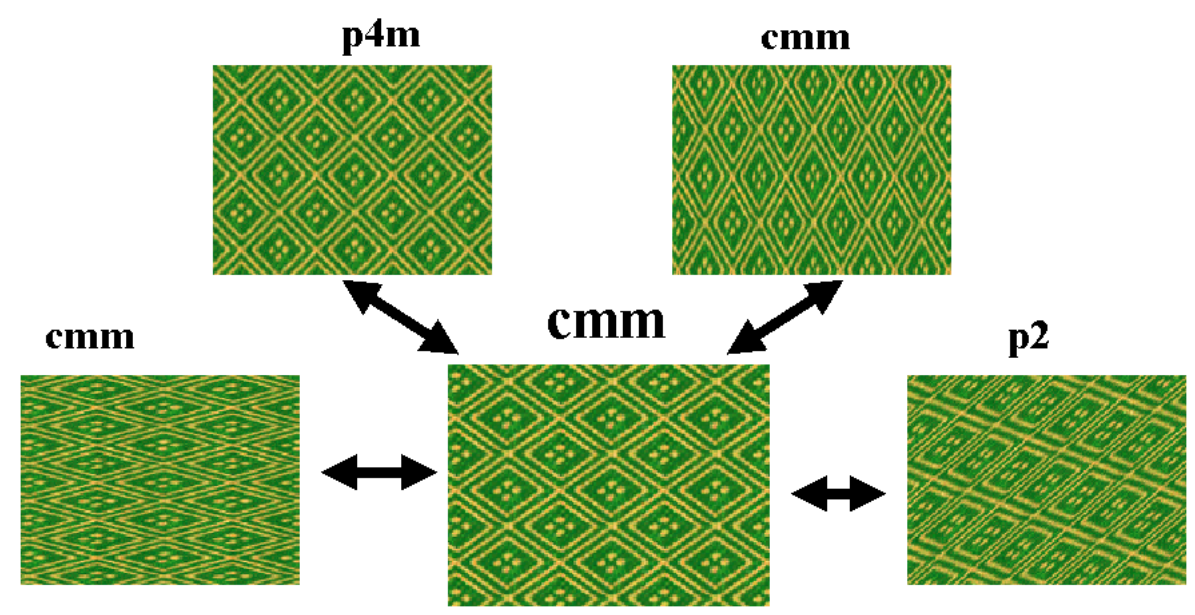

Fig. 4. The original periodic pattern has symmetry group $\mathrm{cmm}$ (middle). Its symmetry group migrates to different groups within its orbit in the wallpaper group transition matrix (Table 2) while the pattern is being affinely transformed.

and $t_{2}$ to $(0, L)$, where $L$ is the larger of the two generating vectors lengths $\left\|t_{1}\right\|$ and $\left\|t_{2}\right\|$.

After transforming to a square lattice, a square generating region (with dimensions $L \times L)$ is cropped from the transformed image. This is used as a template, the rotated and reflected versions of which are correlated with the transformed image to determine what, if any, type of rotation and reflection symmetry it has. In the location determined by the highest correlation peak, a match score between the rotated/reflected template and the image is computed as the mean of the absolute difference between corresponding intensity values. The lower the value of this match score, the more likely it is that the image has that particular rotational/reflectional symmetry. This yields a set of "typical" match scores for that pattern - the mean and standard deviation of these scores are used as an adaptive threshold tailored for this pattern. Match scores associated with rotated/reflected templates are compared to this threshold to determine whether that particular symmetry holds.

An example is shown in Figure 6. The processed values for both square and hexagon lattices are shown below:

\begin{tabular}{c|cccccccc} 
& rot180 & rot120 & rot90 & rot60 & T1 refl & T2 refl & D1 refl & D2 refl \\
\hline square & $\mathbf{0 . 0 4 0}$ & 0.279 & 0.296 & 0.269 & 0.272 & 0.275 & 0.269 & 0.268 \\
hexag & $\mathbf{0 . 0 4 0}$ & $\mathbf{0 . 0 3 8}$ & 0.310 & $\mathbf{0 . 0 4 3}$ & 0.269 & 0.271 & 0.271 & 0.271
\end{tabular}

We find that the pattern only has two-fold rotation symmetry when represented using a square lattice grid, which signifies group $p 2$ (Table 1). To transform the image to a hexagonal lattice structure, the affine transformation is used that 


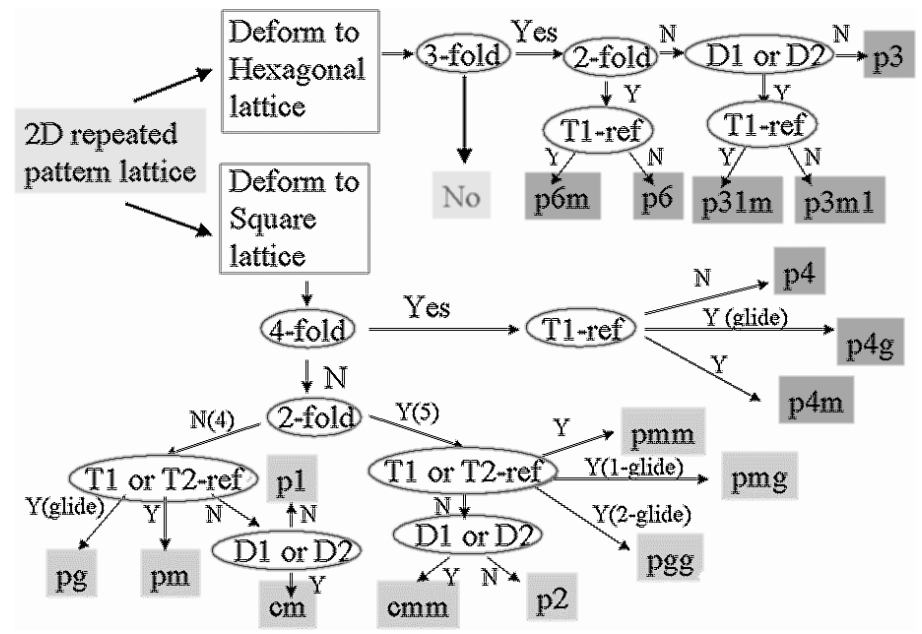

Fig. 5. An algorithm for symmetry group classification of $2 \mathrm{D}$ periodic pattern under affine transformation: $\mathrm{Y}$ (glide) means the reflection symmetry must be a non-trivial glide reflection. $\mathrm{Y}(n) / \mathrm{N}(n)$ means the test result is positive/negative and $n$ is the possible number of symmetry groups need to be further distinguished.

leaves the origin $(0,0)$ fixed while mapping $t_{1}$ to $(L, 0)$ and $t_{2}$ to $(L / 2, L *(\sqrt{3} / 2))$, where $L$ is a length chosen as before. The row labeled "hexag" in the table shows rotation and reflection results for the hexagonally transformed pattern. We see that now, in addition to two-fold symmetry, the pattern also has 60 and 120 degree rotational symmetry. The pattern is uniquely classified as being from the $p 6$ wallpaper symmetry group (Table 1 ). One can also verify this transition between $p 6$ and $p 2$ in the wallpaper group transition matrix (Table 2).

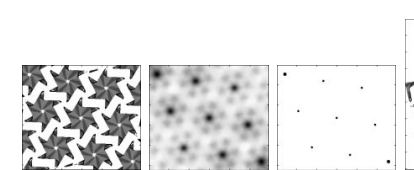

(a)

(b)

(c)

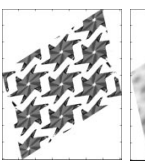

(d)

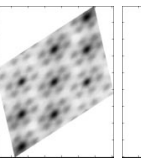

(e)

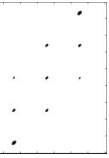

(f)

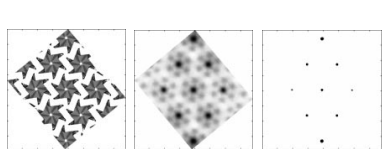

(g)

(h)

(i)

Fig. 6. (a) original image. (b) autocorrelation of image. (c) detected lattice points. (d) transformed image. (e) transformed autocorrelation. (f) transformed lattice points, now a square grid. (g) hexagonal transformed image. (h) transformed autocorrelation. (i) transformed lattice points, now a hexagonal grid. 


\section{Conclusion}

We propose a computational model for periodic pattern perception based on the mathematical theory of crystallographic groups, in particular, the wallpaper groups. This mature mathematical theory provides principled guidelines for analyzing and classifying periodic patterns, and for extracting a patterns' visually meaningful building blocks, namely motifs. This computational model has been implemented and tested on both synthetic and real-world images of periodic patterns. We hypothesize that symmetric tiles form good candidates for human and machine periodic pattern perception.

More importantly, an understanding of the potential symmetry group transitions of a periodic pattern undergoing affine transformation opens a door for us to apply this method to new problems, such as texture perception and re-

placement, localization, robot navigation, and human perceptual organization, among others.

\section{References}

1. E.S. Fedorov. The elements of the study of figures. [Russian] (2) 21. In Zapiski Imperatorskogo S. Peterburgskogo Mineralogichesgo Obshchestva [Proc. S. Peterb. Mineral. Soc.], pages 1-289, 1885.

2. B. Grümbaum and G.C. Shephard. Tilings and Patterns. W.H. Freeman and Company, New York, 1987.

3. Kali. Programs that can automatically generate 2 d planar crystallographic patterns. http://www.geom.umn.edu/apps/kali/.

4. Hsin-Chih Lin, Ling-Ling Wang, and Shi-Nine Yang. Extracting periodicity of a regular texture based on autocorrelation functions. Pattern Recognition Letters, 18:433-443, 1997.

5. Y. Liu and R. T. Collins. A Computational Model for Repeated Pattern Perception using Frieze and Wallpaper Groups. In Computer Vision and Pattern Recognition Conference, June 2000.

6. Y. Liu and R.T. Collins. Frieze and wallpaper symmetry groups classification under affine and perspective distortion. Technical Report CMU-RI-TR-98-37, The Robotics Institute, Carnegie Mellon University, Pittsburgh, PA, 1998.

7. D. Schattschneider. The plane symmetry groups: their recognition and notation. American Mathematical Monthly, 85:439-450, 1978.

8. D.K. Washburn and D.W. Crowe. Symmetries of Culture: Theory and Practice of Plane Pattern Analysis. University of Washington Press, 1991. 